\title{
High Selective Plasma Etching of PMMA to PS
}

\author{
Kazuma Shimomukai, Hiroaki Kawata, Masaaki Yasuda and Yoshihiko Hirai \\ Physics and Electronics, Graduate School of Engineering \\ Osaka Prefecture University, Sakai 599-8531, Japan
}

\begin{abstract}
Polymethylmethacrylate (PMMA) and polystyrene (PS) are important materials for nano pattern fabrication processes such as the directed self-assembly (DSA) lithography. In this paper, the high selective etching of PMMA to PS films is presented by use of $\mathrm{O}_{2}+$ $\mathrm{C}_{4} \mathrm{~F}_{8}$ mixed gas plasmas. Although both etch rates for the PS and PMMA films decrease by increasing the $\mathrm{C}_{4} \mathrm{~F}_{8}$ content, the PS etch rate decreases faster than the PMMA one. The etching selectivity reaches to 6 when the $\mathrm{C}_{4} \mathrm{~F}_{8}$ content is $65 \%$. The aspect ratio of nano pattern is enhanced by use of the high selective PMMA etching. The $250 \mathrm{~nm}$ depth trench pattern of the $80 \mathrm{~nm}$ width is successfully obtained from the $80 \mathrm{~nm}$ depth trench pattern.
\end{abstract}

Keywords: Polymethyl methacrylate, polystyrene, dry etching, $\mathrm{C}_{4} \mathrm{~F}_{8}$

\section{Introduction}

Nanoimprint lithography [1] is superior nanofabrication process because of extremely high resolution and relatively low cost. Polystyrene (PS) and polymethylmethacrylate (PMMA) are very famous resists for the thermal nanoimprint lithography (T-NIL) [2]. Recently, directed self-assembly (DSA) lithography is one of the powerful candidates for nano pattern fabrication around $10 \mathrm{~nm}[3,4]$. A block copolymer of PS and PMMA (PS- $b$-PMMA) is the most popular block copolymer for the DSA lithography. PS and PMMA are very important materials for nano pattern fabrication processes.

It is very important that the PMMA etch rate is larger than the PS one for oxygen plasmas. In the DSA lithography, PS pattern can be obtained by use of the etch rate difference between PMMA and PS resists. The molecular formulas of PS and PMMA are $\left(\mathrm{C}_{8} \mathrm{H}_{8}\right)_{\mathrm{n}}$ and $\left(\mathrm{C}_{5} \mathrm{O}_{2} \mathrm{H}_{8}\right)_{n}$, respectively. Gokan et al. showed that resist etch rates for $\mathrm{O}_{2}$ plasmas greatly depended on the numbers of $\mathrm{C}$ atoms and $\mathrm{O}$ atoms in a monomer unit [5]. Since no $\mathrm{O}$ atoms contain in the monomer unit of PS, the PS etch rate for $\mathrm{O}_{2}$ plasmas is low. The etching selectivity of PMMA to PS films is usually 2 3. Yamamoto showed that the high selective PMMA etching to PS was obtained by CO plasma [6].

In this paper, $\mathrm{C}_{4} \mathrm{~F}_{8}$ is added to $\mathrm{O}_{2}$ plasma in order to obtain a high selective etching of PMMA to PS. $\mathrm{C}_{4} \mathrm{~F}_{8}$ is widely used for $\mathrm{CF}$ film deposition [7]. A resist etch rate is reduced by the film deposition. Since $\mathrm{O}$ atoms contain in the PMMA monomer (internal $\mathrm{O}$ atoms), the deposited film on the PMMA can be partially removed by the internal $\mathrm{O}$ atoms. On the other hand, since no oxygen atoms contain in the PS monomer, no film etching by the internal $\mathrm{O}$ atoms is induced. Therefore, it is expected that the PS etch rate decreases faster than the PMMA one as increasing the $\mathrm{C}_{4} \mathrm{~F}_{8}$ content, and the selectivity of PMMA to PS increases. The selectivity of PMMA to PS is shown for various $\mathrm{C}_{4} \mathrm{~F}_{8}$ contents. Next, the pattern aspect enhancement process is proposed by use of the high selective etching.

\section{Experiment}

2.1 High selective etching of PMMA to PS

For the resist etching, a commercial ICP etcher (ULVAC, NE550) is used in our experiments. The $\mathrm{RF}$ powers to the antenna coil, $\mathrm{P}_{\mathrm{ANT}}$, and the sample stage where samples are placed, $\mathrm{P}_{\mathrm{BIAS}}$, are fixed at $600 \mathrm{~W}$ and $10 \mathrm{~W}$, respectively. $\mathrm{P}_{\text {BIAS }}$ is adjusted to a relatively small value. Since the incident ion energy to samples is mainly controlled by $\mathrm{P}_{\mathrm{BIAS}}$, the incident ion energy is small for low $\mathrm{P}_{\text {BIAS. }}$ Then, the physical sputter etching, which has low material selectivity, must be suppressed. 
PS

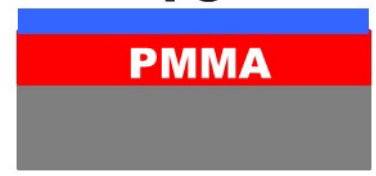

(a)

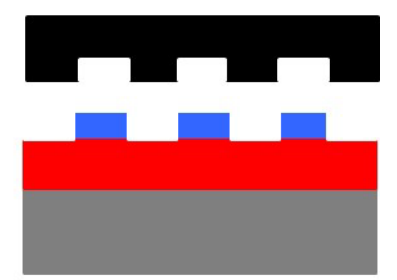

(b)

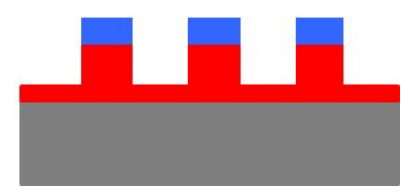

(c)

Fig. 1 Process flow of aspect ratio enhancement process.

We use the mixed gases of $\mathrm{Ar}, \mathrm{O}_{2}$, and $\mathrm{C}_{4} \mathrm{~F}_{8}$. This gas chemistry is widely used for $\mathrm{SiO}_{2}$ selective etching over $\mathrm{Si}$ [8]. $\mathrm{O}_{2}$ is the most commonly used gas for a resist etching. $\mathrm{C}_{4} \mathrm{~F}_{8}$ induces the $\mathrm{CF}$ film deposition, and the etching selectivity of PMMA to PS films can be enhanced. Ar is used for the dilute gas. In our experiments, the Ar flow rate is fixed at $50 \mathrm{sccm}$ and the flow rate of $\mathrm{O}_{2}+\mathrm{C}_{4} \mathrm{~F}_{8}$ mixed gas is always kept to $50 \mathrm{sccm}$.

The etch rates of PMMA and PS films are measured. Cyclohexanone is used as the solvent for PMMA $(\mathrm{Mw}=120 \mathrm{~K})$ and $\mathrm{PS}(\mathrm{Mw}=350 \mathrm{~K})$ resins. The PMMA and PS films are fabricated on a Si wafer. The films are etched by the ICP etcher for $1 \mathrm{~min}$. The film thicknesses before and after the etching are measured, and the etch rates are obtained.

2.2 Aspect ratio enhancement process by the selective etching

Since the etch rate of PS film is smaller than that of PMMA film, PMMA patterns with high aspect ratio can be obtained when PS patterns are used as the PMMA etching mask (aspect ratio enhancement process). The aspect ratio enhancement process is shown in Fig. 1.

(a) The bi-layer film of PS/PMMA is fabricated. The thicknesses of the PS film and the PMMA film are $80 \mathrm{~nm}$ and $1.2 \mu \mathrm{m}$, respectively. (b) The nano pattern of the $80 \mathrm{~nm}$ trench is fabricated to

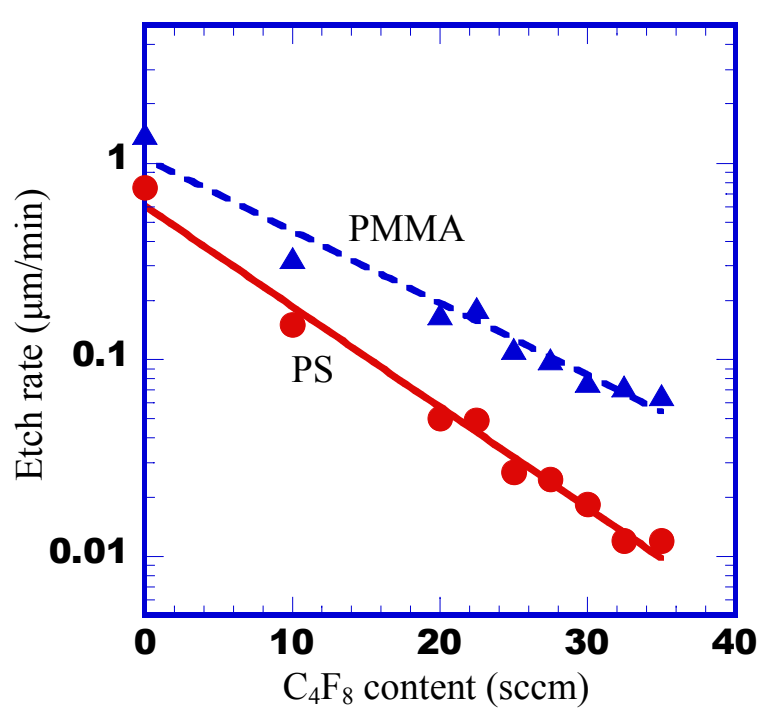

Fig. 2 Relation between PS, PMMA etch rates and $\mathrm{C}_{4} \mathrm{~F}_{8}$ content.

the PS film by T-NIL. The pressure and the temperature are $10 \mathrm{MPa}$ and $140{ }^{\circ} \mathrm{C}$, respectively. c)The PS residual layer is removed (PS residual remove process) and the PMMA film are etched (PMMA etching process).

\section{Results and Discussion}

\subsection{High selective etching of PMMA to PS}

The etch rates of the PMMA and the PS films for various $\mathrm{C}_{4} \mathrm{~F}_{8}$ gas contents are shown in Fig. 2.

The etch rates for both films decrease rapidly by increasing the $\mathrm{C}_{4} \mathrm{~F}_{8}$ content. Note that the etch rate reduction of the PS film is faster than that of the

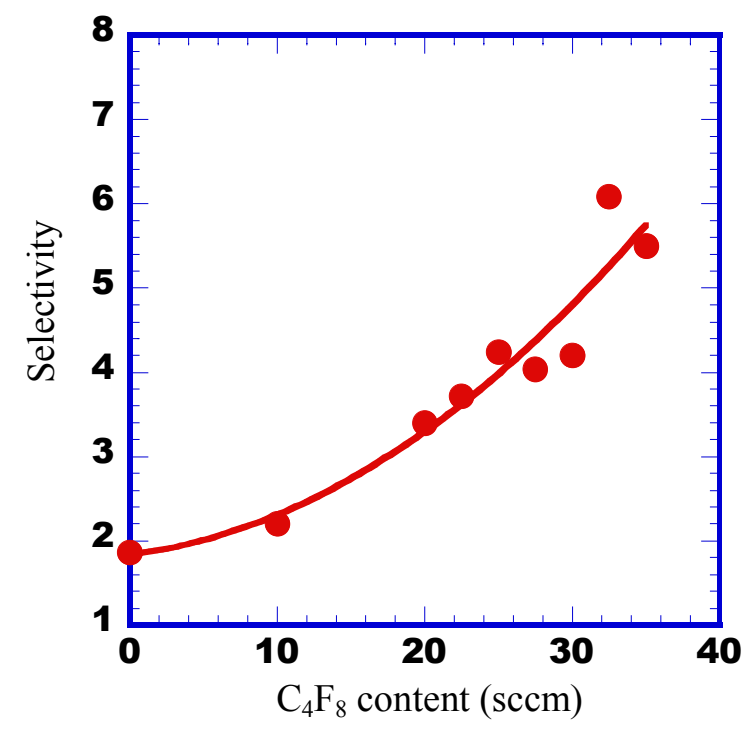

Fig. 3 Relation between selectivity of PMMA to PS and $\mathrm{C}_{4} \mathrm{~F}_{8}$ content. 


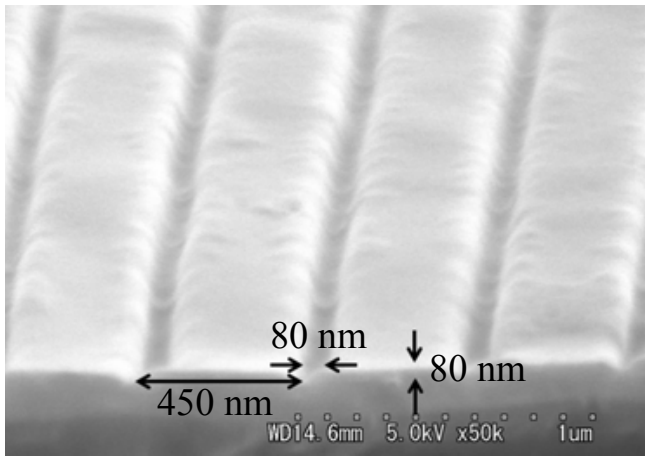

Fig. 4 PS pattern fabricated by NIL
PMMA film. The selectivity, which is the ratio of the PMMA etch rate to the PS one (PMMA/PS), is shown in Fig. 3. For a pure $\mathrm{O}_{2}$ plasma $\left(\mathrm{C}_{4} \mathrm{~F}_{8}\right.$ content $=0 \mathrm{sccm})$, the selectivity is about 2 . The selectivity increases as the $\mathrm{C}_{4} \mathrm{~F}_{8}$ content increases. The selectivity becomes about 6 for the $\mathrm{C}_{4} \mathrm{~F}_{8} / \mathrm{O}_{2}=$ $32.5 \mathrm{sccm} / 17.5 \mathrm{sccm}$.

\subsection{Improvement of aspect ratio of nanopatterns} by the selective etching

Figure 4 shows the PS pattern after T-NIL. The trench width and depth are $80 \mathrm{~nm}$, that is, the aspect ratio is unity. For the PS residual remove process, the $\mathrm{C}_{4} \mathrm{~F}_{8}$ content of $10 \mathrm{sccm}$ is used because the PS film should be etched by a moderate etching speed. Although it is difficult to determine the PS residual layer thickness from Fig. 4 , the etching time is chosen for $10 \mathrm{~s}$. Figure 5 shows the fabricated pattern after the PS residual remove process. Since the trench depth of $120 \mathrm{~nm}$ is larger than the initial PS thickness of $80 \mathrm{~nm}$, the PS residual layer must be removed. After the PS

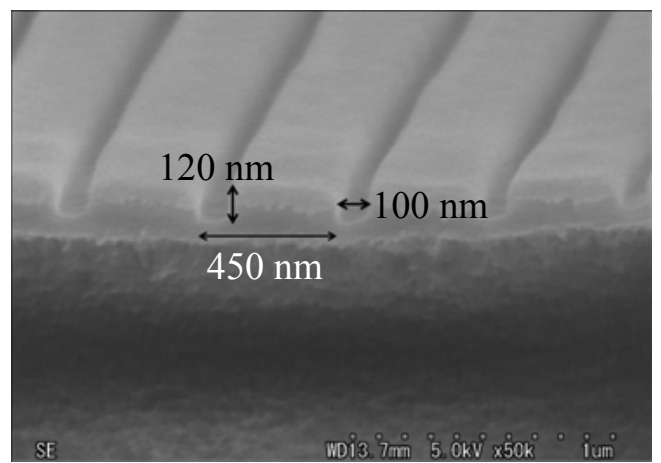

Fig. 5 Fabricated pattern after the PS residual remove process.

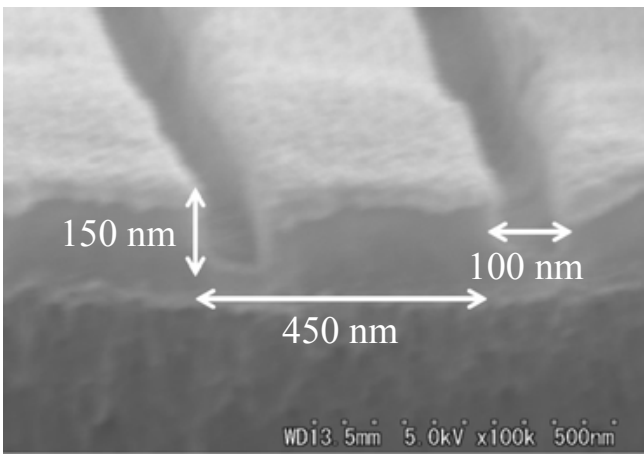

Fig. 6 Fabricated pattern after 60 s PMMA etching process.

residual remove process, the PMMA film is etched by the high selective etching. The gas chemistry of $\mathrm{O}_{2} / \mathrm{C}_{4} \mathrm{~F}_{8}=27.5 \mathrm{sccm} / 22.5 \mathrm{sccm}$ is used for the PMMA etching process. Figure 6 shows the fabricated pattern after the PMMA etching process of $60 \mathrm{~s}$. The trench depth is $150 \mathrm{~nm}$. The trench can be enhanced from that of $120 \mathrm{~nm}$ after the PS residual layer remove process. However, the etching depth during the PMMA etching process is only $30 \mathrm{~nm}$. Since the PMMA etch rate is 180 $\mathrm{nm} / \mathrm{min}$ as shown in Fig. 2, the value of $30 \mathrm{~nm}$ is much smaller than the value expected from the etch rate measurement. This value is similar to the PS etch rate of $50 \mathrm{~nm}$. Figure 7 shows the fabricated pattern after the PMMA etching process of $120 \mathrm{~s}$. It is clear that the trench depth is almost unchanged, but the roughness of PS layer becomes very large.

\subsection{Process with osmium barrier layer}

In our experiments, both the PMMA and PS resins are dissolved by cyclohexanone. It is

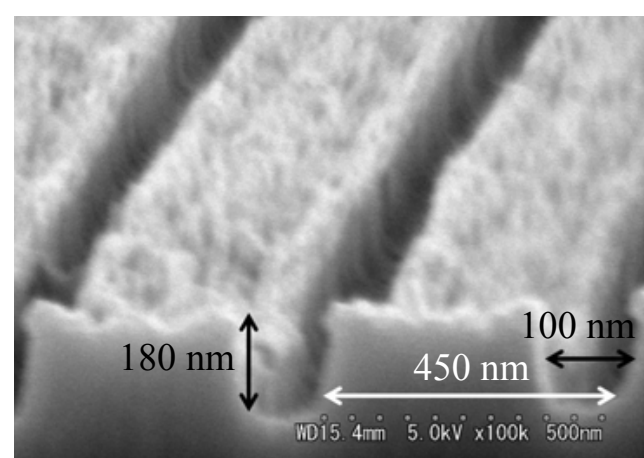

Fig. 7 Fabricated pattern after $120 \mathrm{~s}$ PMMA etching process. 


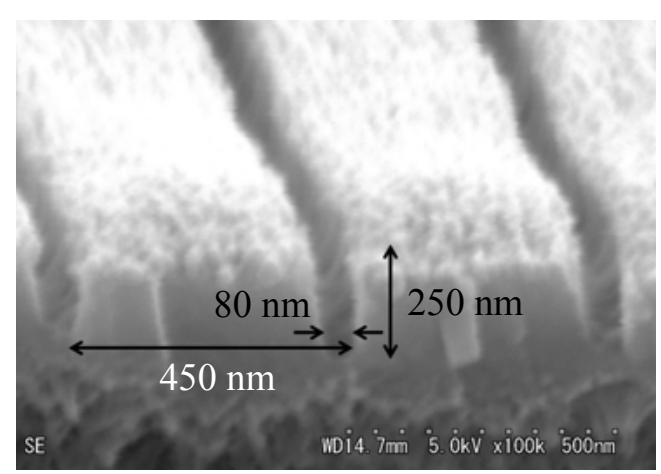

Fig. 8 Fabricated pattern after 120s PMMA etching process with osmium barrier layer.

afraid that the mixing layer of the PMMA and PS resins is formed during the PS and PMMA bi-layer film fabrication. Moreover, the mixing layer may be generated during the T-NIL process, because the imprint temperature is higher than the glass transition temperatures of the PMMA and the PS films. The formation of the mixing layer should be suppressed. A barrier layer between the PMMA and the PS films must be effective in order to avoid the mixing layer formation. Thin osmium film is used as the barrier layer. The osmium film is deposited by a commercial osmium coater for SEM sample preparation (Neoc-pro supplied by Meiwafosis co. ltd). It is considered that osmium film is suitable for the barrier film by the following reasons. No damage must be induced in the osmium film by the PS spin coating, because the osmium film is tough to organic solvents such as cyclohexanone. Since the osmium film can be easily removed by $\mathrm{O}_{2}$ contained plasmas, no additional etching process is necessary. Moreover, the deposition process is easily carried out (the total time for the deposition process is shorter than $10 \mathrm{~min}$ ). The sample preparation process is same as the bi-layer film fabrication except that the osmium deposition process is inserted between the PMMA and PS film fabrication processes. The deposition time of osmium is $10 \mathrm{~s}$. The etching conditions for the PS residual remove and the PMMA etching processes are same as those described in the section 3.2. Figure 8 shows the fabricated pattern after the PMMA etching process of $120 \mathrm{~s}$. The trench width and depth are $80 \mathrm{~nm}$ and $250 \mathrm{~nm}$, respectively. Clearly, the trench depth is deeper than that in Fig. 7 where the etching conditions are same. However, the etching depth is only $100 \mathrm{~nm}$ although the expected PMMA etching depth for $120 \mathrm{~s}$ is $360 \mathrm{~nm}$. The osmium barrier layer is effective for the suppression of the PS and PMMA mixing, but the PS and PMMA mixing cannot be removed completely.

\section{Conclusions}

The PMMA and PS film etch rates are examined for $\mathrm{O}_{2}+\mathrm{C}_{4} \mathrm{~F}_{8}$ mixed gas plasmas in order to obtain the high selective etching of PMMA to PS films. When the $\mathrm{C}_{4} \mathrm{~F}_{8}$ content is increased, the etch rates for both films decreased. The PS etch rate decreases faster than the PMMA one, and the etching selectivity reaches to 6 at $65 \% \mathrm{C}_{4} \mathrm{~F}_{8}$ content. A fine pattern aspect ratio is enhanced by use of the high selective PMMA etching. The 250 $\mathrm{nm}$ depth trench pattern of the $80 \mathrm{~nm}$ width is successfully obtained from the $80 \mathrm{~nm}$ depth trench pattern when osmium barrier layer is used.

\section{References}

1. S. Y. Chou, P. R. Krauss, and P. J. Renstrom, Appl. Phys. Lett. 67 (1995) 3114.

2. H.-C. Scheer, N. Bogdanski, M. Wissen, and S. Möllenbeck, Microelectro. Eng. 85 (2008) 890.

3. D. J. C. Herr, J. Mater. Res., 26 (2011) 122.

4. C. Bencher, J. Smith, L. Miao, C. Cai, Y. Chen, J. Y. Cheng, D. P. Senders, M. Tjio, H. D. Truong, S. Holmes, and W. D. Hinsberg, Proc. SPIE, 7970 (2011) 79700F.

5. H. Gokan, S. Echo, and Y. Ohnishi, J. Electrochem. Soc., 130 (1983) 143.

6. H. Yamamoto, T. Imamura, M. Omura, I. Sakai, and H. Hayashi, Jpn, J. Appl. Phys., 53 (2014) 03DD03.

7. M. A. Blauw, T. Zijlstra, and E. van der Drift, $J$. Vac. Sci. Technol. B, 19 (2001) 2930.

8. M. Matsui, T. Tatsumi, and M. Sekine, J. Vac. Sci. Technol. A, 19 (2001) 2089. 